Int. J. Dev. Biol. 61: 451-457 (2017)

doi: $10.1387 / \mathrm{ijdb} .170053 \mathrm{bc}$

\title{
Role of Mad2 expression during the early development of the sea urchin
}

\author{
ODILE BRONCHAIN ${ }^{1}$, WAEL JDEY ${ }^{2}$, LAETITIA CARATY ${ }^{3}$ and BRIGITTE CIAPA*,1 \\ ${ }^{1}$ Paris-Saclay Institute of Neuroscience, CNRS, Univ. Paris-Sud, Université Paris-Saclay, ${ }^{2}$ Institut Curie, UMR \\ CNRS 3347 and ${ }^{3}$ Institut de Génétique et Microbiologie, UMR 8621, Université Paris XI, Orsay, France
}

\begin{abstract}
Mitotic arrest deficient 2 (Mad2) belongs to the spindle assembly checkpoint (SAC), a mechanism that blocks progression of the cell cycle until microtubule attachment to kinetochores is complete. It has been found to be involved in the resistance of cancer cells to "anti-mitotic" drugs such as paclitaxel. Mad2 controls meiotic progression, but its role during sea urchin development had never been investigated. Furthermore, the existence of a SAC in this species had never been proved. The present data show that a Mad2 protein, highly homologous to that of humans, is expressed in this species. Mad2 expression increases during development, becoming confined to the endomesoderm at gastrula stages. The level of Mad2 expression is enhanced in embryos that do not gastrulate after treatment with anti-mitotic drugs, lithium or inhibition of the ERK pathway. Mis-aligned and lagging chromosomes were induced after injection of an anti-Mad2 antibody or a Mad2 morpholino. Our results point to the role of a non-canonical SAC involving Mad2 in the control of mitotic divisions of the sea urchin embryo.
\end{abstract}

KEY WORDS: Mad2, spindle assembly checkpoint, mitosis, sea urchin, antimitotics

Mad2 belongs to the spindle assembly checkpoint (SAC) that blocks progression of cell cycles until microtubule (MT) attachment to kinetochores is complete. Depletion of Mad2 usually accelerates the metaphase-anaphase transition and perturbs mitosis in various cultured cell types. In contrast, Mad2 overexpression induces a variety of mitotic alterations in various types of cells and is a commonly observed event in human cancers (Lischetti and Nilsson, 2015). Mad2 is essential for mouse development from meiotic progression (Touati and Wassmann, 2016) to gastrulation (Dobles et al., 2000). However, Mad2-null Drosophila are viable and fertile and cellular division occurs properly despite having no SAC (Buffin et al., 2007).

Drugs affecting MTs such as paclitaxel and vinblastine trigger activation of the SAC leading to cell cycle arrest and ultimately cell death. These are extensively used in cancer treatments and are referred to "anti-mitotics". Nocodazole and colchicine also affect MTs but their toxicities have curtailed their development as anticancer agents. Acquired resistance is a crucial issue for the therapeutic use of most anti-mitotics, a process likely linked to alterations in Mad2 expression (Bargiela-Iparraguirre et al., 2014).
We considered whether the sea urchin model could be useful to assess Mad2 expression dynamics. Treatment of sea urchin embryos with anti-mitotics during the first mitosis inhibits division (Sluder et al., 1997, Strickland et al., 2005), but the effect of long time treatments has not been investigated. This study was necessary bound to the question concerning the existence of a SAC mechanism, which is still debated in this species (Sluder et al., 1997). The present data suggest that Mad2 belongs to a non-canonical SAC, a system that involves the ERK cascade and controls mitosis as previously reported (Ciapa and Philippe, 2013). Since Mad2 expression has been correlated with epithelial-mesenchymal transition (EMT), a process used by cells that metastasize (Nakaya and Sheng, 2013), and that both EMT and Mad2 expression are altered by "anti-mitotic" drugs, we propose that the sea

Abbreviations used in this paper: $\mathrm{Ab}$, antibody; $\mathrm{APC}$, anaphase promoting complex; EMT, epithelial-mesenchymal transition; ERK, extracellular signal-regulated kinases; GRN, gene regulatory network; Mad2, mitotic arrest deficient 2; MT, microtubule; MAPK, mitogen-activated protein kinase; NEB, nuclear envelope breakdown; SAC, spindle assembly checkpoint; WISH, whole mount in situ hybridisation.

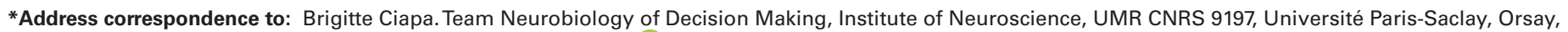
France.Tel.: (33) 01691568 09. E-mail: brigitte.ciapa@u-psud.fr (iD http://orcid.org/0000-0002-3794-6209
}

Supplementary Material (one figure and one table) for this paper is available at: http://dx.doi.org/10.1387/ijdb.170053bc

Submitted: 1 March, 2017; Accepted: 27 April, 2017.

ISSN: Online 1696-3547, Print 0214-6282 
Homo sapiens S purpuratas P lividus

Homo sapiens $\mathrm{S}$ purpuratus P lividus

Homo sapiens $S$ purpuratas P lividus

Homo sapiens $S$ purpuratus P lividus

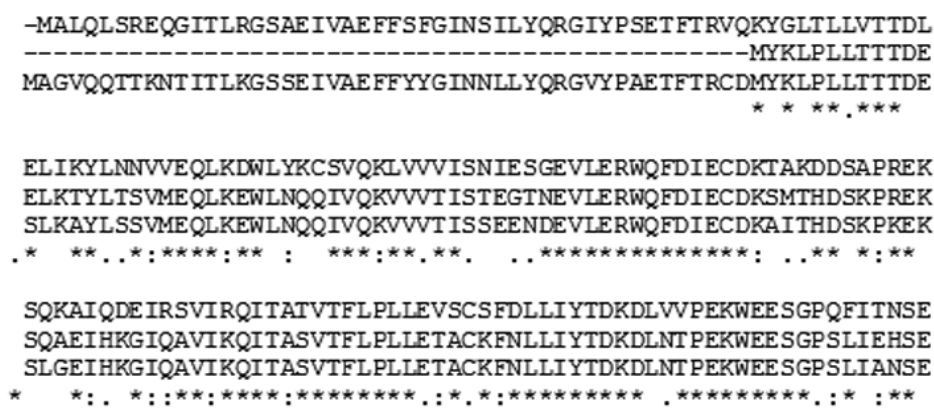

EVRLRSFTTT I HKVNSMVAYKI PVND

EVRLRSFTTSIHKVDAMVSYKLMDGI

EVRLRSFTTS IHKVDAMVSYKLMDGI

$* * * * * * * * *: * * * *:::^{*}: * *:$.
Fig. 1. Mad2 sequence of $\boldsymbol{P}$. lividus. Comparison with S. purpuratus and human (Homo sapiens). Alignment was performed though the Clustal Omega program. Positions are labelled as explained in http://Www.ebi.ac.uk/Tools/msa/ clustalo/help/faq.html\#20. urchin embryo could be useful to study how MT-disrupting agents affect Mad2 expression.

\section{Results}

\section{Expression of mitotic arrest deficient-2 (MAD2) during development}

The sequence of the translated Mad2 protein is highly similar to SP-MAD2A of S. purpuratus (SPU_016042) and to that of human (Fig.1). This allowed us to use a commercial anti-Mad2 Ab raised against the human protein. IF stainings (Fig.2) show that Mad2 is minimally expressed in the unfertilized egg (t0), then its expression increases in the fertilized embryo $(1 \mathrm{~h})$ to remain the same in each blastomere at least to the 16 cell stage (not shown). By the blastula stage (20h), Mad2 is uniformly expressed. However at the gastrula stage (28h), only the vegetal pole shows a faint expression (Fig.2A, 28h b and b'). These results corroborated those obtained by WISH (Fig.2B, 28h b and b'). Therefore, Mad2 expression increases during development and is predominantly found in the endomesoderm at the gastrula stage.

Vegetalization of the embryo after treatment with $20 \mathrm{mM} \mathrm{LiCl}$ (Poutska et al., 2007) or 5 MM U0126 (Röttinger et al., 2004) expands Mad2 expression territory as it does for various genes showing a similar early expression pattern (Poutska et al., 2007).
WISH staining shows a strong Mad2 expression over the entire vegetilized embryo (Fig.3A). This is confirmed by RT-PCR analysis (Fig.3B). Mad2 expression increases with the time of $\mathrm{LiCl}$ treatment (Fig.3Ba). A $2.7+/-0.5(n=6)$ and $1.8+/-0.4(n=5)$ fold increase in Mad2 expression was measured in embryos treated for $28 \mathrm{~h}$ with $20 \mathrm{mM} \mathrm{LiCl}$ or $5 \mu \mathrm{M}$ U0126, respectively (Fig. 3Bb).

\section{Role of Mad2 during development}

Unfertilized eggs from four different batches were injected with a Mad2-Ab as a blocking reagent, fertilized and then allowed to develop. None of 59 injected eggs from two different batches divided. In the two others, 26 out of 34 injected eggs failed to divide. These embryos showed diverse mitotic alterations (Fig.4) at the 4-cell (Fig.4A) or 8-cell stages (Fig.4B) compared to control embryos, such as chromosomes lagging (Fig.4Aa-C), abnormal metaphase plate (Fig.4B1a-b) or aberrant chromosome arrangements (Fig.4B2a-b). Eggs that could divide once remained blocked at the 2-cell stage with an aberrant chromosome segregation (Fig.4B3a-C). $95 \%$ of the non-injected embryos or embryos injected with a control antibody (anti-presenilin $2 \mathrm{Ab}$ raised against a sequence without similarity found in the sea urchin genome) divided normally, indicating a specific effect of the anti-Mad2 Ab (not shown).

We then injected a Mad2-Mo as a complementary knockdown strategy. A rescue experiment was performed by co-injecting the

A
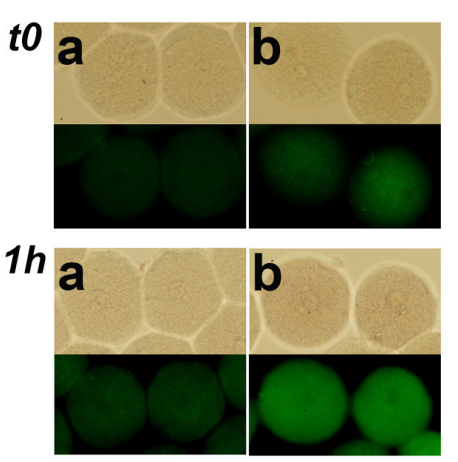

B

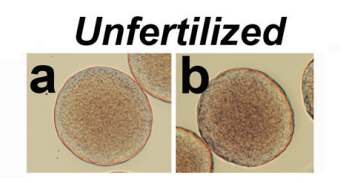

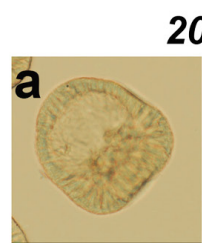

$20 h$
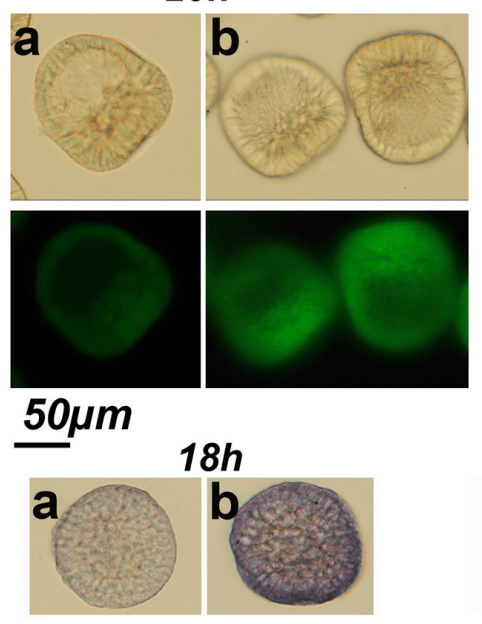

$28 h$
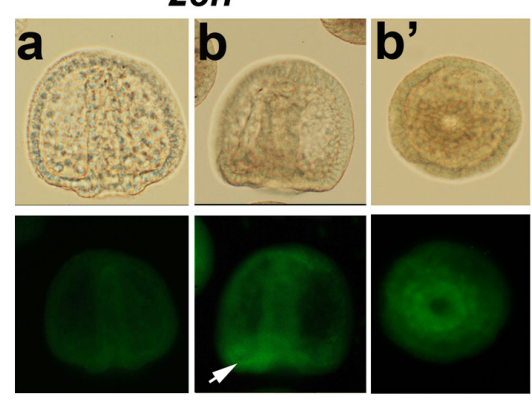

$28 h$

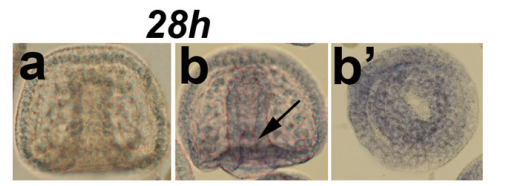

Fig. 2. Mad2 expression during development. (A) IF labelling. Transmitted light (upper panels) and IF images (lower panels) in control conditions (second Ab only, a) or after labelling with the anti-Mad2 Ab (b and $\left.b^{\prime}\right)$. (B) WISH. Control hybridisation using a sense probe (a) or an antisense probe (b and $\left.b^{\prime}\right)$. A view from the side of the archenteron of gastrula $\left(b^{\prime}\right)$ is shown in $(A, B)$. 
A

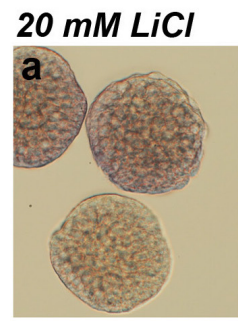

$5 \mu M \cup 0126$

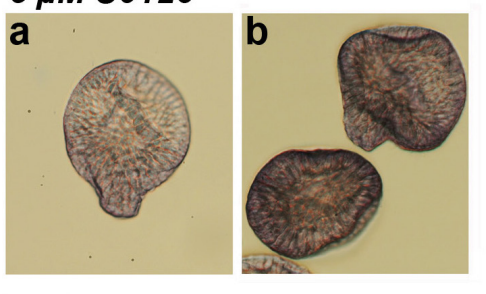

a

$50 \mu m$

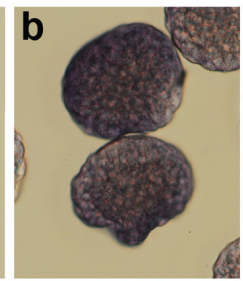

b

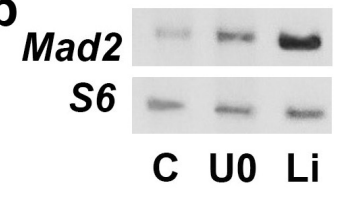

B

A

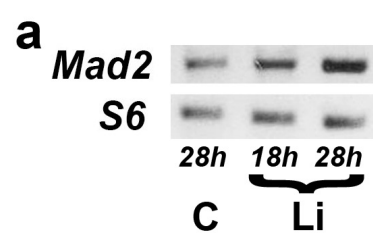

Fig. 3 (Left). Extension of the Mad2 expression territory after vegetalization with $\mathbf{2 0} \mathbf{~ m M ~ L i C l ~ o r ~} \mathbf{5} \mathbf{~ m M ~ U 0 1 2 6 . ~ ( A ) ~ W I S H . ~ E m b r y o s ~ t r e a t e d ~ a r - ~}$ rested $28 \mathrm{~h}$ post-fertilization. Embryos are labelled with sense (a) or antisense (b) probes. Untreated embryos are as those shown in Fig.2B-28 $h$. (B) Semi-quantitative RT-PCR. Expression of S6 is used as an internal control. Transcription of Mad2 increases with the time of treatment with $20 \mathrm{mM}$ LiCl (a) and is higher in 28h embryos treated with this amount of LiCl or with $5 \mathrm{mM} \mathrm{U0126} \mathrm{(b)} \mathrm{than} \mathrm{that} \mathrm{of} \mathrm{non-treated} \mathrm{embryos} \mathrm{(C).}$

Fig. 4 (Right). Effect of anti-Mad2 Ab injection. (A) Embryos arrested at time of the control 4-cell stage. Light microscopy (a), Hoechst staining (b) and visualization of injected embryos (c). A $4 x$ enlargement of lagging chromosome is shown (inset ${ }^{*}$ in b). (B) Typical images (1, 2 and 3) of embryos arrested at the control 8-cell stage. Panel 3 show a control embryo (left) and an injected embryo (right) showing one blastomere $(*)$ containing two lots of chromosomes at anaphase (b) with a normal bipolar splindle excluding carboxyfluorescein (c) and a second blastomere from which chromosomes appear to "escape" (arrow).

Mad2-Mo with the Mad2 mRNA. Hoechst labelling was then performed when non-injected embryos reached the 8-16 cells stage. Results obtained from 4 different batches of eggs (Exp1-4) are summarized in Fig.5A and representative images shown in Fig.5B and $\mathrm{C}$. All embryos injected with the control Mad2-Mc divided normally (Fig.5B).

Injection of the Mad2-Mo induced mitosis arrest in all batches of eggs at a time that varied across batches (Fig.5A). In Exp1 and Exp2, more than $60 \%$ of eggs did not divide at all. In Exp3 and Exp4, 10-26\% embryos divided three times, giving 8-16 cells embryos without obvious alterations. The remaining embryos showed an aberrant number of dividing blastomeres with alterations in chromosome segregation. In all batches, all embryos injected with the Mad2-Mo did not reach a normal 32-cell stage and all died $5 \mathrm{~h}$ to $6 \mathrm{~h}$ following fertilization (not shown). The rescue experiment was conducted on eggs from Exp.3/4 (Fig.5C). Injection of the Mad2-mRNA alone had no effect (Fig.5C-1) while aberrant chromosome segregation occurred in a high percentage in eggs injected with the Mad2-Mo (Fig.5A and Fig.5C-2). Co-injection of the Mad2-mRNA and the Mad2-Mo (rescue) increased the \% of third division obtained after injection of the Mad2-Mo only (Fig.5A), resulting in more than $50 \%$ embryos able to progress normally to the 16-cells stage (Fig.5C-3). Similar alterations were observed upon anti-Mad2 Ab injection, although slightly less pronounced/ delayed phenotypes appearing (Fig.4).

In order to test whether Mad2-Mo injection impacts Mad2 synthesis, eleven embryos were injected with Mad2-Mo and then immunostained for Mad2 (Fig.5D). Seven embryos showed obvi- ous altered development, containing 4-8 blastomeres of various sizes. In these, the Mad2 staining level varied from $75 \%$ to $20 \%$ of that measured in 2 or 4 cell stage control embryos from the same experiment (not shown) as seen in Fig.5.

Unfertilized eggs seem therefore to contain a maternal pool of Mad2, the level of which varies across batches. This may be sufficient to accomplish the first three mitotic divisions but a de novo synthesis is required to sustain long term development.

\section{Effect of anti-mitotic drugs on Mad2 expression}

A dose-response assay was performed by adding, $10 \mathrm{~min}$ post-fertilization, nocodazole, colchicine, vinblastine or paclitaxel at various concentrations up to $5 \mu \mathrm{M}$. Three batches of eggs were tested and gave similar results. Fig. $6 \mathrm{~A}$ shows the developmental chronology. Nocodazole had a maximal effect at $1 \mu \mathrm{M}$, a concentration at which embryos remained at NEB until they begun dying $7 \mathrm{~h}$ after fertilization, the entire population being dead at 22h (Fig.6B). A $5 \mu \mathrm{M}$ treatment with paclitaxel, colchicine or vinblastine did not alter the first divisions (Fig. $675 \mathrm{~min}, \mathrm{C}, \mathrm{D}$ and E) but delayed development and induced various alterations from the morula (Fig.6 7h, D and E, egg a) to early blastula stages (Fig.6 7 h, C and D, egg b). Blastulas were hollow, elongated (Fig.6 22h, C, D and $E$ ) and deprived of primary mesenchyme cells (PMCs). These embryos either hatch (Fig. $622 \mathrm{~h}, \mathrm{C}$ both eggs, D and E egg b) or not (Fig. 6 22h, D and E, egg a). When control embryos reached gastrulation, treated ones were either still at blastula stage (Fig.6 30h, C, D and E, egg a) or began to fragment (Fig.6C, D and E, $30 \mathrm{~h}$ egg b). Few attempted gastrulation (Fig.6 30h, $\mathrm{C}$ egg c) but 


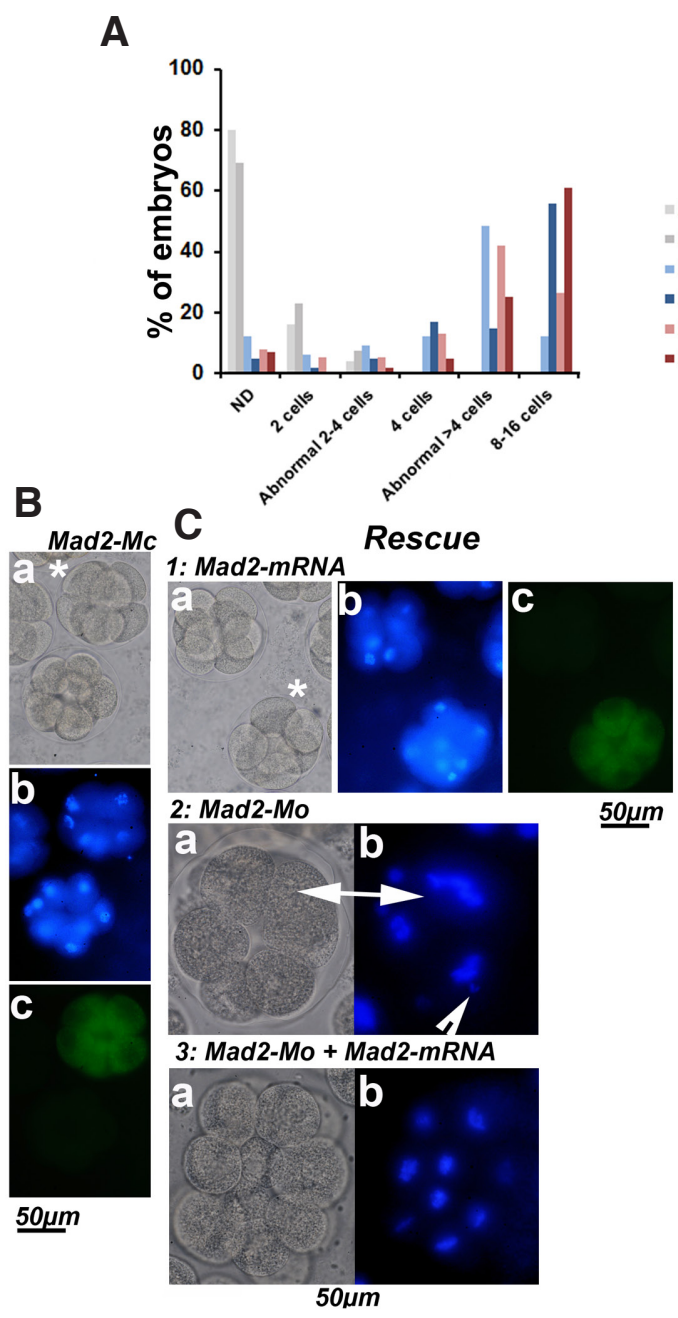

Fig. 5. Effect of Mad-Mo injection. (A) Quantification of cell divisions in embryos arrested at the 8-16 cell stage in four independent batches (Exp. 1-4) and injected either with the Mad2-Mo (all exp.) or with both Mad2-Mo and Mad2-mRNA (rescue, Exp 3 and 4). Injected embryos were counted as not divided (ND), "2 cells," "4 cells" or "8-16 cells." They were further scored "abnormal" when obvious aberrant chromosome segregation and/or altered number and/or size of blastomeres was detected. $n=$ total number of injected embryos per experiment. (B,C) Observations by light microscopy (a), after Hoechst (b) or carboxyfluorescein (c) stainings showing injected eggs $(*)$. B: Injection with the Mad2-Mc $\left.{ }^{*}\right)$. C: rescue experiment. Injection of the Mad2-mRNA alone (1); (2): Injection with the Mad2-Mo showing obvious aberrant chromosome segregation (double arrow). (3): Co-injection of the Mad2-Mo with Mad2-mRNA. Injections in images 2 and 3 were confirmed by carboxyfluorescein staining (not shown). (D) Mad2 immunostaining of a Mad2-Mo injected embryo. The injected embryo $\left(^{*}\right)$ contains an abnormal number of cells (a), a reduced level of Mad2 immunostaining compared to the non-injected embryo (b) and mis-segregation of chromosomes (arrow, c).

all eventually died $10 \mathrm{~h}$ later (not shown).

Mad2 expression in treated embryos was further assayed except in nocodazole treated embryos that do not divide. Compared to control blastula (Fig.7Ab), the IF level increased after treatment with $5 \mu \mathrm{M}$ of paclitaxel (Fig.7Bb), colchicine (Fig.7Bb) or vinblastine (Fig.7Cb). A similar increase in Mad2 transcription is seen by WISH studies (Fig.8A). While only the vegetal pole is stained in non-treated embryos at the gastrula stage, treated embryos are strongly and uniformly stained. This finding was confirmed by RTPCR performed on four different batches (Fig.8B). It must be pointed that the sensitivity to each drug varies across batches (Fig.8Bb).

Finally, treatment with these anti-mitotic drugs affected ERK phosphorylation. $1 \mu \mathrm{M}$ nocodazole arrested the first cell cycle (Fig.6B) without altering P-ERK inactivation (not shown). In the three different embryos batches, P-ERK levels increased significantly in embryos treated with vinblastine and paclitaxel compared to controls (Fig.9A,B).

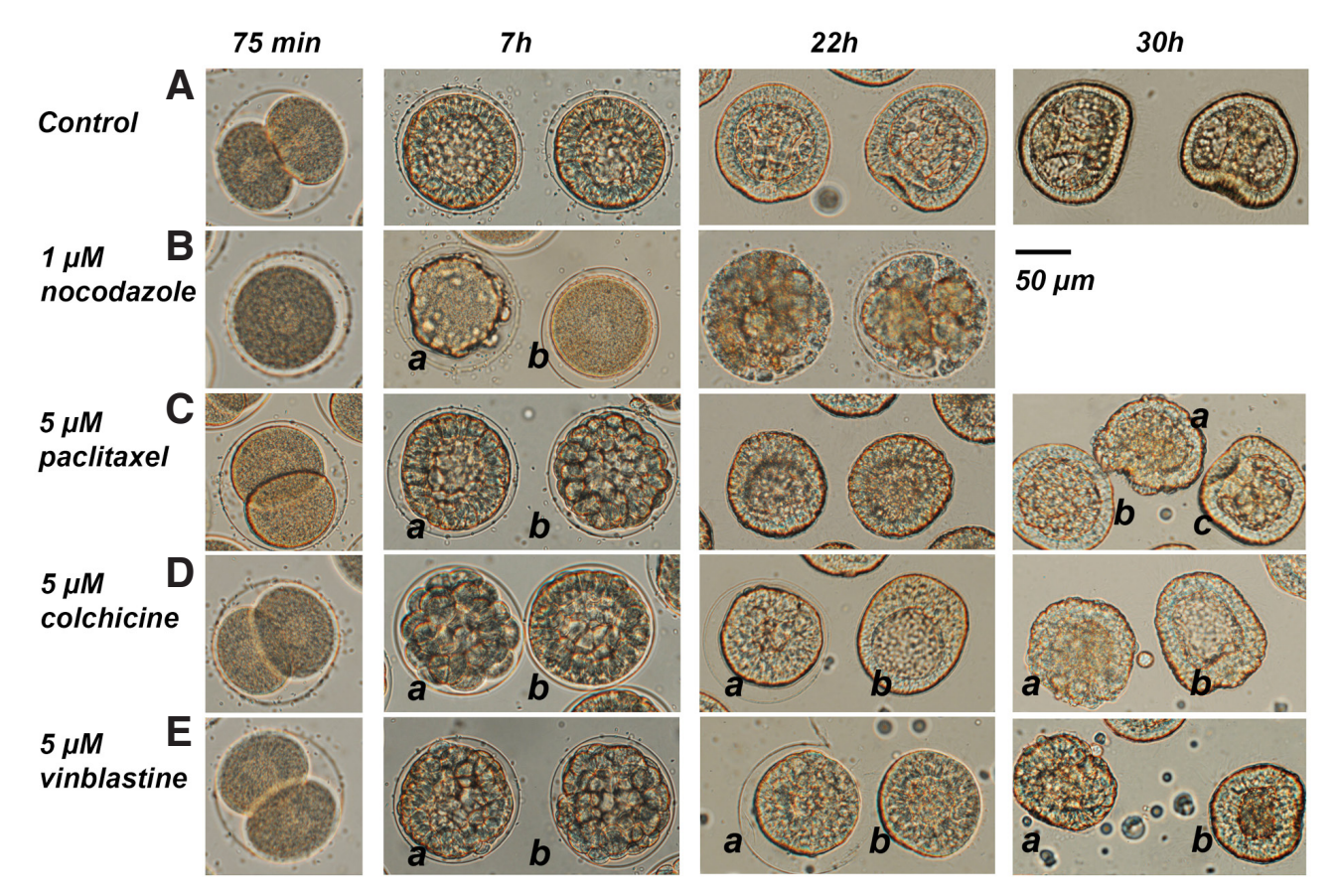

\section{Discussion}

Several results indicate that Mad2 transcription and translation occur at early developmental stages in sea urchins. Indeed, Mad2cDNAclones can be found in the $P$. lividus library generated from unfertilized eggs (PI_EGG) or gastrula stages (PI_GASPLU). Our IF, WISH and RT-PCR experiments also support this early expression. We failed to detect Mad2 by WB analysis, which may be due to Mad2 ability to form dimers (Lischetti and Nilsson,

Fig. 6. Observation by transmitted light microscopy of early development after treatment with the anti-mitotic drugs. (A) Normal chronology in control embryos: first mitotic divisions (75 mins), early blastula (7h), early (22h) and late gastrula (30h). (B) Effect of $1 \mathrm{mM}$ nocodazole. (C,D,E) Effect of $5 \mathrm{mM}$ paclitaxel, colchicine or vinblastine, respectively. 


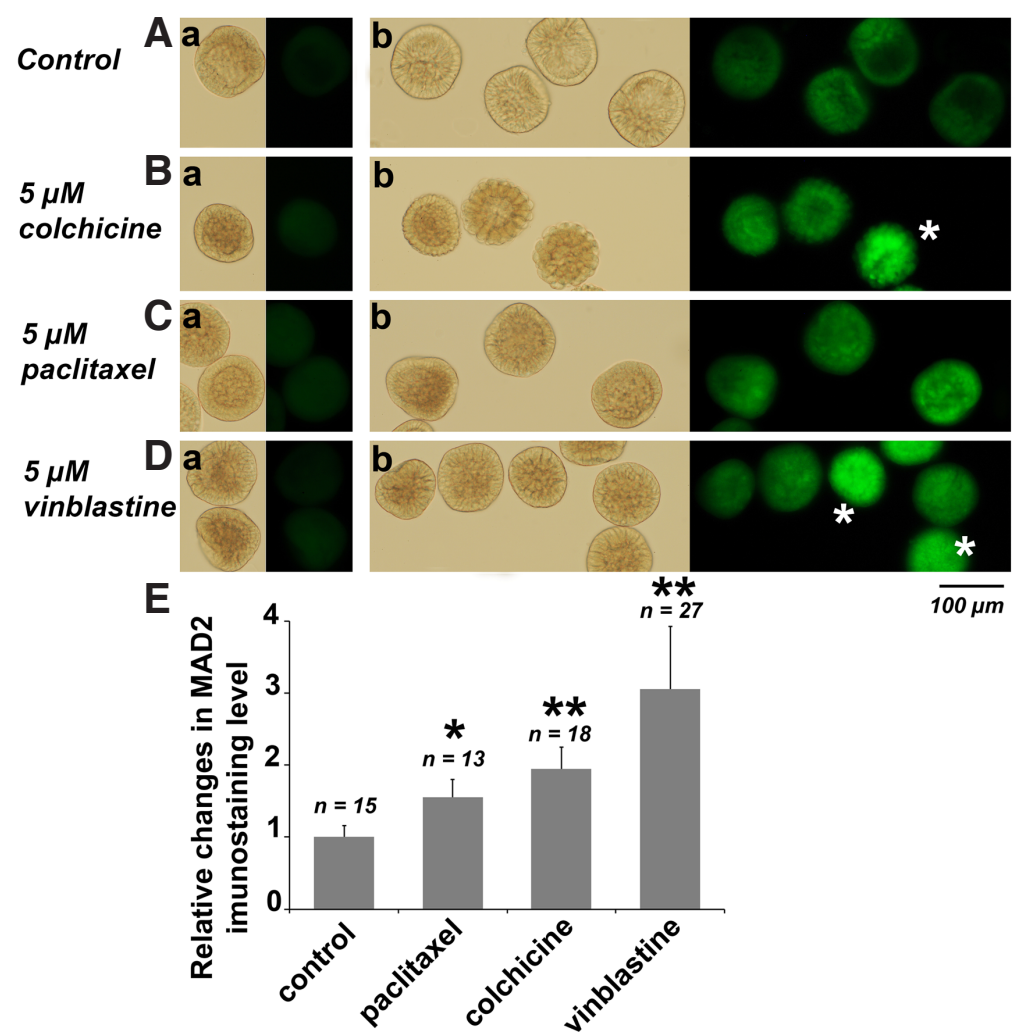

Fig. 7. Expression of Mad2 at the blastula stage (18 $\mathrm{h}$ postfertilization) after treatment with the antimitotic drugs. Control with the 2nd Ab only (a) and IF staining (b) with transmitted light microscopy (left panel) and IF (right panel) images. (A) Non-treated embryos. (B,C,D) Embryos treated with $5 \mathrm{mM}$ colchicine, vinblastine or paclitaxel, respectively. Some embryos are highly fluorescent (stars). (E) Quantification of IF signals as shown in $(A, B, C, D)$. Results are mean + - sem of IF level recorded in $n$ embryos.

the idea of a "non-canonical SAC", different from the mammalian one, in the sea urchin embryo as proposed over the last years (Sluder et al., 1997). This idea was introduced in Giardia intestinalis where Mad2 knockdown also stops mitosis (Vicente and Cande, 2014).

The maternal amount of the SAC components stocked in oocytes before fertilization may decrease during oocyte aging as reported in mammalians (Touati and Wassmann, 2016). This would define a starting SAC efficiency that could account for variation across egg batches.

Mad2 is mostly expressed in the endomesoderm and overexperssed in vegetelized embryos. This could be expected as cellular division becomes confined to the endomesoderm and parts of the oral ectoderm after gastrulation (Zhang et al., 2015). It would be interesting to analyse the efficiency of $S A C$ at later stages.

Our results suggest that anti-mitotic drugs act within a similar range of concentration throughout the embryo, allowing gastrulation under a given threshold. This is not

2015). The anti-Mad2 Ab we used may only label native form(s) that are bound or not to kinetochores and may diffuse in the cytoplasm as reported in rat oocytes (Zhang et al., 2004). This could account for the uniform Mad2 expression that we observed by IF until the blastula stage.

Mad2 depletion by Ab or morpholino injections leads to misaligned and lagging chromosomes and blocks division. This phenotype is expected after SAC activation but not inhibition. How to interpret these results? Mad2 and Bub3 are both expressed in sea urchin and highly homologous to human ones. In contrast, Mad1 and BubR1 are far less conserved and only one Aurora kinase is expressed in the sea urchin $S$. purpuratus (Table 1, S1). Our data reinforce

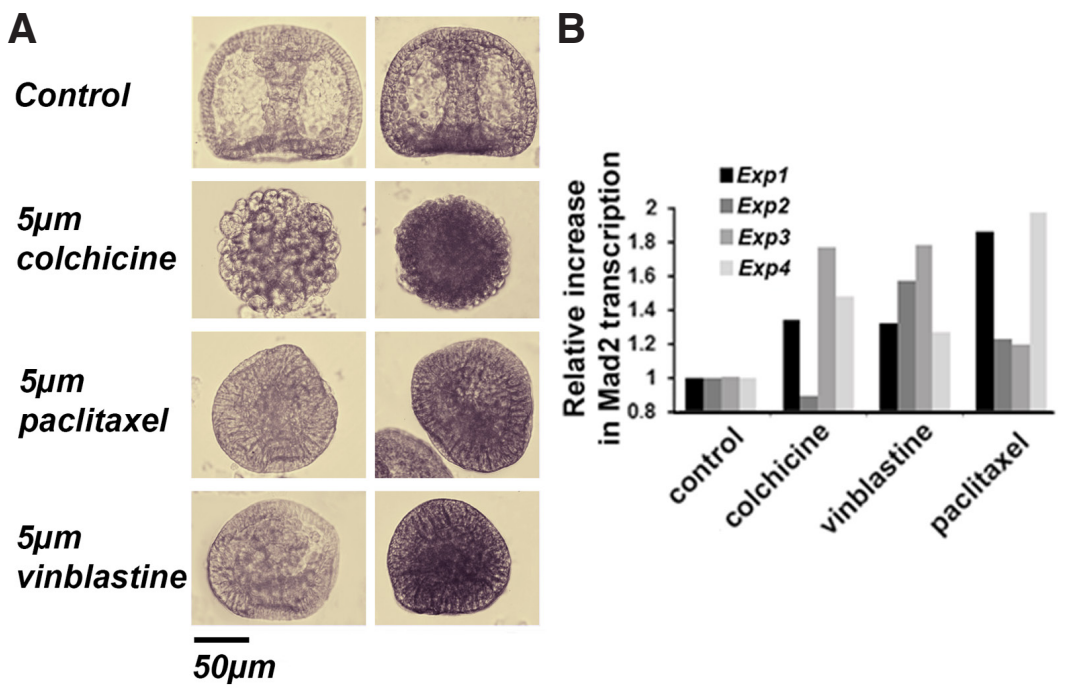
surprising since MTs control EMT during gastrulation (Stephens et al., 1989). However, gastrulation failure may also be due to other processes requiring MT function. Finally, the delayed development upon $6 \mathrm{hr}$ of treatment may be due to mechanisms unrelated to the SAC itself as these drugs could interfere with proteins that bind MTs. This is the case for Disheveled (Dsh) that binds MTs and controls the Wnt pathway which establishes the animal-vegetal axis (Torres and Nelson, 2000).

No DNA fragmentation was detected in embryos treated with the antimitotic drugs even when displaying obvious phenotypic manifestations (S2). Such difficulty to assess DNA fragmentation has been reported (Houel-Renault et al., 2013). Finally, the sensitivity to each drug varies across embryo batches. As discussed above, this may be due to a variety of parameters including seasonal heterogeneity, time of gametes retention in the gonads etc. For example, Cameron etal., (1993) reported a "considerable batchto-batch variability" in the frequency of gastrula arrest that occurs after injection of (5HT-R) mRNA into sea urchin blastomeres.

Fertilized sea urchin eggs treated with nocodazole did not form any spindle and did not show increased level of ERK phosphorylation, contrary to fertilized eggs that arrest at entry in mitosis in acidic ASW containing

Fig. 8. Transcription of Mad2 in $28 \mathrm{~h}$ embryos treated or not with $5 \mathrm{mM}$ colchicine, paclitaxel or vinblastine. (A) WISH. Sense (control, left panel) or antisense (right panell probes. (B) Quantification in 4 different batches of embryos (Exp 1-4) of semi-quantitative RT-PCR performed as explained in Fig. $3 B$ 


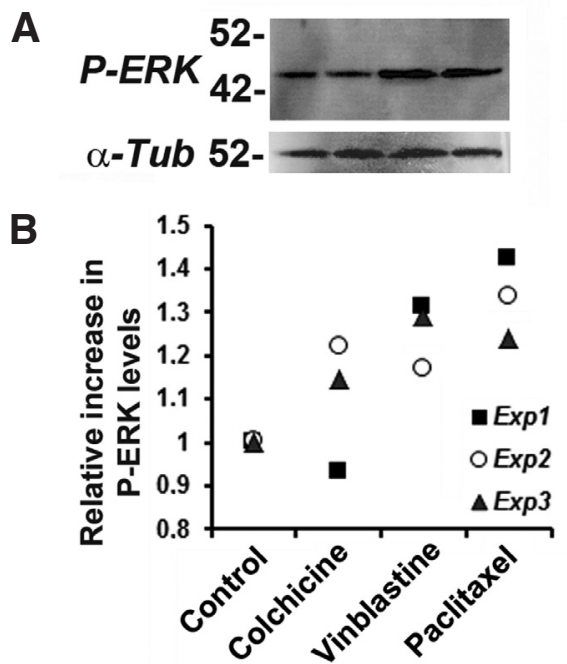

Fig. 9. Expression of P-ERK in $28 \mathrm{~h}$ embryos treated or not with $5 \mathrm{mM}$ colchicine, vinblastine or paclitaxel. (A) Anti-P-ERK (upper panel) and anti-a-Tub (lower panel) WBs. (B) Relative changes in P-ERK level after quantification of 3 experiments performed as shown in A (Exp.1).

acetate with a very high level of P-ERK (Ciapa and Philippe, 2013). Obviously, different means of arresting the first mitotic divisions do not stimulate a common pathway. The diversification of ERK roles during development (Röttinger et al., 2006) may explain the increase in ERK phosphorylation seen at later stages in embryos treated with the antimitotic drugs.

Mechanisms that modify Mad2 expression in cancer cells are not understood. The gene regulatory networks described in this model are the best understood among animal models, particularly that regulating gastrulation (Hinman and Cheatle Jarvela, 2014), which requires the process of EMT. The study of Mad2 expression in the sea urchin embryo may help understanding how it is altered by antimitotic drugs, controlled by the MEK/ERK cascade or modified during the EMT associated with gastrulation, a crucial step of development that involves sets of events common to those described in cells that metastasize (Nakaya and Sheng, 2013).

\section{Materials and Methods}

Manipulation of the Paracentrotus lividus gametes, Western blot (WB), immunofluorescence (IF) labelling, microscopy observations and quantification of WB bands were performed as previously described (Houel-Renault et al., 2013). Mad2 immunostaining level was quantified by Image $\mathrm{J}$ and normalized by comparison to the value determined in control eggs and/or blastomeres arbitrarily taken as 1 .

Stock solutions of nocodazole $(25 \mathrm{mM})$, paclitaxel $(10 \mathrm{mM})$, vinblastine $(10 \mathrm{mM})$ and colchicine $(10 \mathrm{mM})$ were prepared in DMSO. Vegetalization was induced by transferring embryos in ASW containing $20 \mathrm{mM} \mathrm{LiCl} \mathrm{(1M}$ stock solution in distilled water) or $5 \mu \mathrm{M}$ U0126 (Calbiochem, $15 \mathrm{mM}$ stock solution in DMSO) after the first mitosis had occurred and leaving them to develop in these solutions.

We used a phospho- p42/44 ERK Ab (\#9106, Cell Signaling Tech), an anti- $\alpha$ tubulin Ab (\#CP06, Calbiochem), an anti-Mad2 antibody (Biolegend,\#602101) with an anti-rabbit FITC Ab (Jackson), an anti-presenilin 2 (H76, sc-7861, Santa Cruz Tech) and an anti-tubulin Ab YL1/2 (Chemicon) with an anti-rat FITC IgG (Jackson ImmunoResearch) to label spindles.

To obtain an in situ Mad2 probe, we used a clone (PKOAAA6YG11RM1) from a Paracentrotus lividus cDNA library (http://octopus.obs-vlfr.fr). The translated sequence of the insert was transferred in pBluescript II KS+ using the pair of primers Mad2-F (5'-cgcggatccCGGGTGTACAACAAACAACGAA-3') and Mad2-R (5'- cccaagcttTCCCATCCATGAGTTTGTAAGACA 3'). We used BLASTP (http://www.ncbi.nlm.nih.gov/BLAST/) to compare sequences. WISH probes were synthesized and labelled with digoxigenin11-UTP (Roche) following a protocol described by Duboc et al., (2005).

For semi-quantitative RT-PCR, total RNA was purified from dry pellets with an RNeasy Plus Micro Kit (Qiagen). RT was performed using the SuperScript II RT-kit (Invitrogen) and reverse (RV) primers (see below) of both Mad2 and S6 (used as internal control). S6 sequence was retrieved from the same Paracentrotus lividus cDNA library. PCR was performed with a GoTaq G2 Flexi DNA Polymerase-kit (Promega) with couples of RV and forward (FW) primers of either Mad2 or S6. Bands were quantified by Image J, normalized by comparison to $\mathrm{S} 6$ and then expressed relative to the value of control embryos taken as 1. Primers are as follows:

FW-Mad2: AAGGCATACCTGTCATCGGTAATGG

RV-Mad2: AAGTCCTTGTCGGTGTAAATGAGCA

FW-S6: GATGCGTTGAAGAGGTCACTTACTG

RV-S6: GATGTCACGACGGAAGTACTCGTTT

Injections were conducted as previously described (Houel-Renault et al., 2013) with a 1/10 dilution of the anti-Mad2 Ab stock solution, $0.15 \mathrm{mM}$ Mad2-Morpholinos (Mad2-Mo and Mad2-Mc) and/or $0.15 \mu \mathrm{g} / \mu \mathrm{l}$ Mad2 mRNA which is in the same range than RNA amounts injected by others (Duboc et al., 2005). All were diluted in distilled water containing $2 \mathrm{mM}$ carboxyfluorescein to visualize injection.

The Mad2-Mo (TCGTTGTTTGTTGTACACCCGCCAT), which targets a region 120 nucleotides 5 ' to the putative start codon, and a standard negative control morpholino (Mad2-Mc, CCTCTTACCTCAGTTACAATTTATA), which targets a human beta-globin intron mutation) were obtained from Gene Tools.

Synthetic mRNA was synthesised as follows: The entire Mad2 ORF was amplified from the same clone used for ISH as described above and using the following primers: 5'-CGGAATTCCATGTACAAGCTACCCCTGCTAAC-3'and 5'-CCGCTCGAGTTATATCCCATCCATGAGTTTGTAAG-3' and subcloned into the pCS2FLAG vector (www.addgene.org/16331/) at the EcoRI/Xhol restriction sites. Synthetic mRNA was synthesised with a mMachine kit (Life technologies).

No carboxyfluorescein was used when Mad2 immunostaining was performed on embryos injected with the Mad2-Mo. Embryos were treated with ATAZ, fertilized, deposited on a polylysine petri dish labelled with a grid in order to identify the injected eggs, and then microinjected between 10 and 30 mins after sperm addition. IF was performed after fixation of embryos as described above.

Cell death was detected on PFA fixed embryos using a kit (Roche, Cat. No 11684795 910). A positive control was performed as described by the manufacturer.

\section{Acknowledgements}

We thank C Billam for correcting our manuscript.

\section{References}

BARGIELA-IPARRAGUIRRE J1, PRADO-MARCHAL L, PAJUELO-LOZANO N, JIMÉNEZ B, PERONA R, SÁNCHEZ-PÉREZ I (2014). Mad2 and BubR1 modulates tumourigenesis and paclitaxel response in MKN45 gastric cancer cells. Cell Cycle 13: 3590-3601.

BUFFIN E, EMRE D, KARESS RE (2007). Flies without a spindle checkpoint. Nat Cell Biol 9: 565-572.

CAMERON RA, SMITH LC, BRITTEN RJ, DAVIDSON EH (1994). Ligand-dependen stimulation of introduced mammalian brain receptors alters spicule symmetry and other morphogenetic events in sea urchin embryos. Mech Dev 45: 31-47.

CIAPA B, PHILIPPE L (2013). Intracellular and extracellular pH and $\mathrm{Ca}$ are bound to control mitosis in the early sea urchin embryo via ERK and MPF activities. PLoS One 8: e66113.

DOBLES M, LIBERAL V, SCOTT ML, BENEZRA R, SORGER PK (2000). Chromo- 
some missegregation and apoptosis in mice lacking the mitotic checkpoint protein Mad2. Cell 101: 635-645.

DUBOC V, RÖTTINGER E, LAPRAZ F, BESNARDEAU L, LEPAGE T (2005). LeftRight Asymmetry in the Sea Urchin Embryo is Regulated by Nodal Signaling on the Right Side. Dev Cell 9: 147-158.

HINMAN VF, CHEATLE JARVELAAM (2014). Developmental gene regulatory network evolution: insights from comparative studies in echinoderms. Genesis 52: 193-207.

HOUEL-RENAULTL,.PHILIPPE L, PIQUEMALM, CIAPAB (2013). Autophagy is used as a survival program in unfertilized sea urchin eggs that are destined to die by apoptosis after inactivation of MAPK1/3 (ERK2/1). Autophagy 9(10): 1527-1539.

LISCHETTI T, NILSSON J (2015). Regulation of mitotic progression by the spindle assembly checkpoint. Mol Cell Oncol 2: e970484.

NAKAYA, Y, SHENG G (2013). EMT in developmental morphogenesis. Cancer Lett 341: 9-15.

POUSTKA AJ, KÜHN A, GROTH D, WEISE V, YAGUCHI S et al., (2007). A global view of gene expression in lithium and zinc treated sea urchin embryos: new components of gene regulatory networks. Genome Biol 8: R85.

RÖTTINGER E, BESNARDEAU L, LEPAGE T (2004). A Raf/MEK/ERK signaling pathway is required for development of the sea urchin embryo micromere lineage through phosphorylation of the transcription factor Ets. Development 131:1075-1087
SLUDER G, THOMPSON EA, MILLER FJ, HAYES J, RIEDER CL (1997). The checkpoint control for anaphase onset does not monitor excess numbers of spindle poles or bipolar spindle symmetry. J Cell Sci. 110: 421-429.

STEPHENS L, HARDIN J, KELLER R, WILT F (1986) The effects of aphidicholin on morphogenesis and differentiation in the sea urchin embryo. Dev Biol 118: 64-69.

STRICKLAND LI, DONNELLY EJ, BURGESS DR (2005). Induction of cytokinesis is independent of precisely regulated microtubule dynamics. Mol Biol Cell 16 : 4485-4494.

TORRES MA, NELSON WJ (2000). Colocalization and redistribution of Dishevelled and actin during Wnt-induced mesenchymal morphogenesis. J Cell Biol 149: 1433-1442.

TOUATI SA, WASSMANN K (2016). How oocytes try to get it right: spindle checkpoint in meiosis. Chromosoma 125: 321-335.

VICENTE JJ, CANDE WZ (2014). Mad2, Bub3, and Mps1 regulate chromosome segregation and mitotic synchrony in Giardia intestinalis, a binucleate protist lacking an anaphase-promoting complex. Mol Biol Cell 25: 2774-2787.

ZHANG M, KOTHARI P, LAMPSON MA (2015). Spindle Assembly Checkpoint Acquisition at the Mid-Blastula Transition. PLoS One 10: e0119285.

ZHANG D, MA W, LI YH, HOU Y, LI SW, MENG XQ, SUN XF, SUN QY, WANG WH (2004). Intra-oocyte Localization of MAD2 and Its Relationship with Kinetochores, Microtubules, and Chromosomes in Rat Oocytes During Meiosis. Biol Reprod 71: 740-748. 
Further Related Reading, published previously in the Int. J. Dev. Biol.

Unc-5/netrin-mediated axonal projection during larval serotonergic nervous system formation in the sea urchin, Hemicentrotus pulcherrimus Kouki Abe, Tomoko Katow, Shioh Ooka and Hideki Katow

Int. J. Dev. Biol. (2013) 57: 415-425

https://doi.org/10.1387/ijdb.120256hk

\section{Genomic control of patterning}

Isabelle S. Peter and Eric H. Davidson

Int. J. Dev. Biol. (2009) 53: 707-716

https://doi.org/10.1387/ijdb.072495ip

Aurora-A: an expedition to the pole of the spindle in Xenopus egg extracts. Jacek Z. Kubiak and Claude Prigent.

Int J Dev Biol. (2016) 60: 255-261.

Oocyte ageing and its cellular basis.

Ursula Eichenlaub-Ritter.

Int J Dev Biol (2012) 56: 841-852.

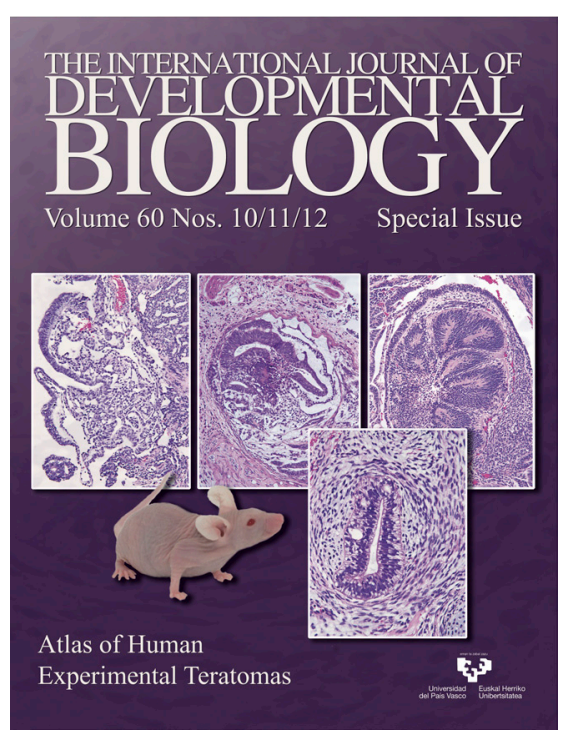

5 yr ISI Impact Factor $(2013)=2.879$

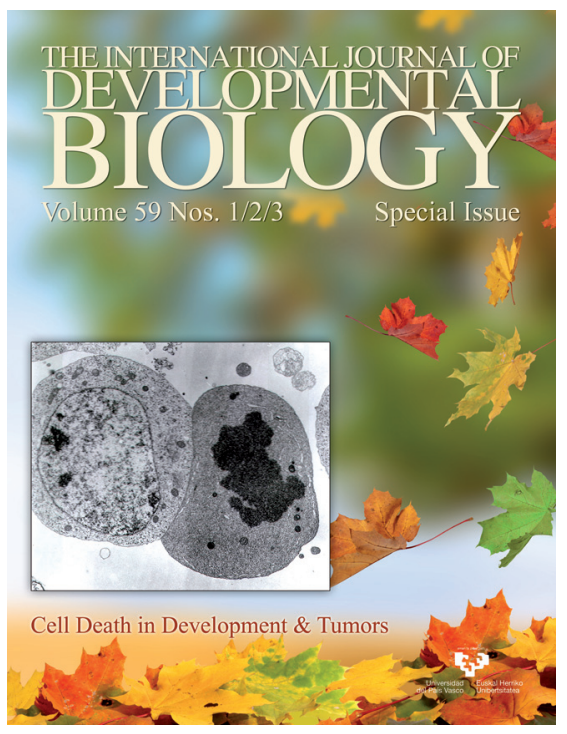

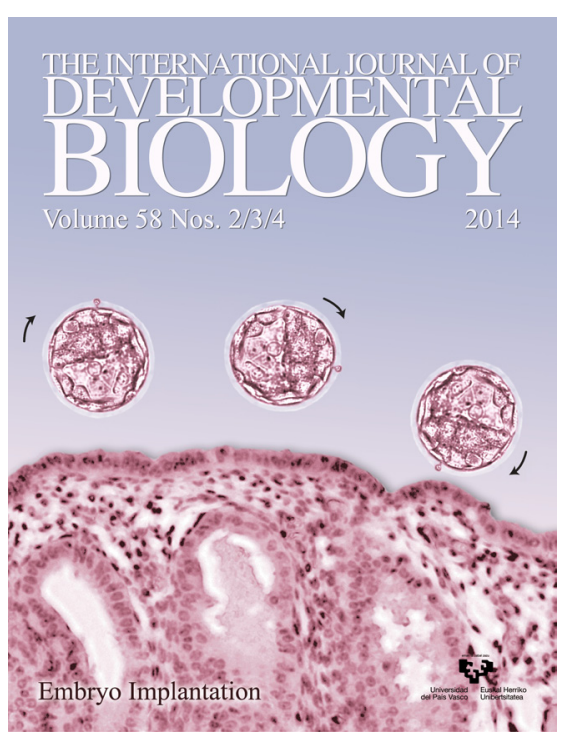

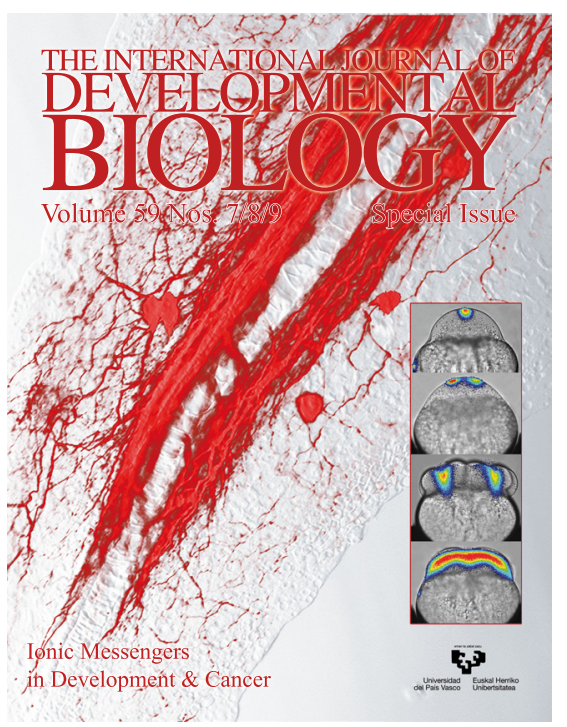

